\title{
Bioeconomics of Sustainable Harvest of Competing Species: A Comment
}

\author{
TIBOR NEUGEBAUER \\ University Hannover, Germany \\ BEGOÑA CASINO \\ University of Valencia, Spain
}

\begin{abstract}
We refer to Flaaten's (JEEM 1991, pp. 163-80) study on competing species. In Theorem 5 (Theorem 6), Flaaten claims that a higher price (harvesting costs) of one species yields a lower (greater) own stock-size and a greater (lower) stock-size of the competing species in the steady state. It is shown that both claims are wrong.
\end{abstract}

Key words Renewable resources, fishery management, multiple species model.

JEL Classification Codes D62, D99, Q22.

\section{Introduction}

Growth of a fishery population is determined to a large extent by its environment. Since one species forms only part of a complex ecological system including prey, competitors, and predators, modeling its dynamics with a single differential or difference equation neglects such ecological interdependence and can lead to incorrect assessments. In the literature, it was pointed out that spillovers occurring from one population into another might crucially change the optimal extraction plan in a fishery (Clark 1976; Flaaten 1991). In contrast to the single species model, the optimal solution can involve harvesting of some species at a loss, as a lower stock increases the profits from the fishery of the interdependent species.

One important paper in this literature is by Flaaten (1991) which studies the bioeconomics of Gause's (1969) deterministic competing species model. In the paper, Flaaten (1991) provides six theorems, of which the last two involve general conjectures on the impact of market parameter changes on long-run stock levels. Theorem 5 states that a higher price of one species will yield a lower own stock level and a greater stock level of the competing species in the steady state. Theorem 6 states that higher harvesting costs of one species will yield a greater own stock level and a lower stock level of the competing species in the steady state. Both theorems rely on the same proof, which turns out to be flawed, as Flaaten acknowledges in an unpublished note. In this paper, we show that the theorems are not general results, but that the prediction of Theorem 5 is even reversed if costless harvesting is assumed.

Tibor Neugebauer is an assistant professor at University of Hannover, Wirtschaftswissenschaftliche Fakultät, Institut für Finanzmarkttheorie, Königsworther Platz 1, D - 30167 Hannover, Germany, email: T.Neugebauer@mbox.vwl.uni-hannover.de. Begoña Casino is an associate professor at the University of Valencia, Facultad de Economia, Av. de los Naranjos, 46022 Valencia, Spain, email: Begona.Casino@uv.es. 
In the remainder of the paper, we review the competing species model and derive the optimal harvesting decision of the sole owner. We follow closely the steps of both Flaaten's unpublished note and Flaaten (1991). We show where the proof of the theorems is flawed and provide a counter example. Finally, we sum up.

\section{The Model}

In the notation, we follow Flaaten (1991). The growth function of species $i$ is given by the logistic production function:

$$
G_{i}\left(X_{1}, X_{2}\right):=r_{i} X_{i}\left(1-X_{i}-\alpha_{i} X_{j}\right),(i, j=1,2, i \neq j)
$$

where $X_{i} \in[0,1]$ denotes the stock of species $i$ relative to its carrying capacity, $\alpha_{i}$ is the dimensionless competition parameter, and $r_{i}$ is the intrinsic growth rate. Throughout the paper, we will assume that $\alpha_{i} \in(0,1), i=1,2$. This is a necessary condition for having an interior, positive stable steady state without harvesting (see Flaaten 1991, p.166).

In contrast to the single species model, the competition parameter is incorporated to describe by how much the living space of species $i$ is affected through the presence of competing species $j$. The greater the competition parameter, the "flatter" the curve of the growth function of species $i$, and the lower the species' stock level in the biological equilibrium without harvesting.

As Flaaten, we only consider selective harvesting; i.e., each unit of effort, $E_{i}$, can be dedicated either to catching species $i=1,2$, but not to both at the same time. The catch rates are $y_{i}\left(X_{i}\right):=E_{i} X_{i}(i=1,2)$. Given Schaefer's harvest function and assuming constant costs per unit effort, the unit harvesting costs are $c_{i}\left(X_{i}\right)=$ $c_{i} / X_{i}(i=1,2) .{ }^{1}$ Under the assumption of constant prices $p_{1}$ and $p_{2}$ of species 1 and 2 , respectively, the total profit from harvesting the two species is:

$$
\pi\left(p_{1}, p_{2}, X_{1}, X_{2}, y_{1}, y_{2}\right):=b_{1}\left(p_{1}, X_{1}\right) y_{1}+b_{2}\left(p_{2}, X_{2}\right) y_{2},
$$

where $b_{i}\left(p_{i}, X_{i}\right):=p_{i}-c_{i}\left(X_{i}\right)(i=1,2)$ denotes the net profit per unit harvest of species $i$.

The management problem of the competing species problem involves the maximization of the present value $(P V)$ of the total profit from both fisheries subject to the two equations of motion. Thus, the externality of the production of species 1 is internalized in the production of species 2 , and vice versa. The planner faces the following two-control problem:

$$
\begin{gathered}
\max _{y_{1}, y_{2}} P V=\int_{0}^{\infty} e^{-\delta t}\left[b_{1}\left(p_{1}, X_{1}\right) y_{1}+b_{2}\left(p_{2}, X_{2}\right) y_{2}\right] d t \\
\text { s.t. } \dot{x}_{i}(t)=G_{i}\left[X_{1}(t), X_{2}(t)\right]-y_{i}(t), i=1,2 \\
0 \leq y_{i}(t) \leq X_{i}(t),
\end{gathered}
$$

\footnotetext{
${ }^{1}$ The unit cost of harvesting species $i$ accounts for $i$ 's catchability quotient.
} 
where $\delta$ denotes the discount rate. The stock sizes change in time according to growth and catch. Assuming an interior solution, the following equation pair determines the steady state optimal solution to the planner's maximization problem: ${ }^{2}$

$$
\begin{aligned}
& b_{1}\left(p_{1}, X_{1}^{*}\right)=1 / \delta\left[b_{1}\left(p_{1}, X_{1}^{*}\right) G_{11}\left(X_{1}^{*}, X_{2}^{*}\right)+b_{2}\left(p_{2}, X_{2}^{*}\right) G_{21}\left(X_{1}^{*}, X_{2}^{*}\right)-c_{1}^{\prime}\left(X_{1}^{*}\right) G_{1}\left(X_{1}^{*}, X_{2}^{*}\right)\right] \\
& b_{2}\left(p_{2}, X_{2}^{*}\right)=1 / \delta\left[b_{1}\left(p_{1}, X_{1}^{*}\right) G_{12}\left(X_{1}^{*}, X_{2}^{*}\right)+b_{2}\left(p_{2}, X_{2}^{*}\right) G_{22}\left(X_{1}^{*}, X_{2}^{*}\right)-c_{2}^{\prime}\left(X_{2}^{*}\right) G_{2}\left(X_{1}^{*}, X_{2}^{*}\right)\right]
\end{aligned}
$$

where $G_{i j}\left(X_{1}^{*}, X_{2}^{*}\right):=\partial G_{i} / \partial X_{j}^{*}(i, j=1,2)$ is the partial derivative of species $i$ 's growth function with respect to the stock of species $j$, evaluated at the steady-state solution. ${ }^{3}$ In the steady state, the total profit of the marginal unit extracted from the stock (on the left-hand side) must equal the discounted marginal rent of this unit (on the right-hand side), which reflects the present value of all future losses that result through harvesting from a lower stock. In the steady state, growth equals catch, $y_{i}=G_{i}\left(X_{1}^{*}, X_{2}^{*}\right)$. We define the steady-state profit function as follows:

$$
\tilde{\pi}\left(p_{1}, p_{2}, X_{1}, X_{2}\right):=\pi\left[p_{1}, p_{2}, X_{1}, X_{2}, G_{1}\left(X_{1}^{*}, X_{2}^{*}\right), G_{2}\left(X_{1}^{*}, X_{2}^{*}\right)\right],
$$

and rewrite equation (4) using $\left(2^{*}\right)$ to obtain:

$$
b_{i}\left(p_{i}, X_{i}\right)=\frac{1}{\delta} \frac{\partial \tilde{\pi}\left(p_{1}, p_{2}, X_{1}, X_{2}\right)}{\partial X_{i}}(i=1,2)
$$

Flaaten's (1991) comparative statics analysis regarding price and cost parameters sets out at equation (5). Following his steps, we differentiate with respect to $p_{1}$ to yield the following:

$$
\begin{gathered}
b_{1 p}+b_{1 x} \frac{\partial X_{1}^{*}}{\partial p_{1}}=\frac{1}{\delta}\left(\frac{\partial^{2} \tilde{\pi}}{\partial X_{1} \partial p_{1}}+\frac{\partial^{2} \tilde{\pi}}{\partial X_{1}^{2}} \frac{\partial X_{1}^{*}}{\partial p_{1}}+\frac{\partial^{2} \tilde{\pi}}{\partial X_{1} \partial X_{2}} \frac{\partial X_{2}^{*}}{\partial p_{1}}\right) \\
b_{2 x} \frac{\partial X_{2}^{*}}{\partial p_{1}}=\frac{1}{\delta}\left(\frac{\partial^{2} \tilde{\pi}}{\partial X_{2} \partial p_{1}}+\frac{\partial^{2} \tilde{\pi}}{\partial X_{2} \partial X_{1}} \frac{\partial X_{1}^{*}}{\partial p_{1}}+\frac{\partial^{2} \tilde{\pi}}{\partial X_{2}^{2}} \frac{\partial X_{2}^{*}}{\partial p_{1}}\right),
\end{gathered}
$$

where:

$$
b_{i p}:=\frac{\partial b_{i}(\cdot)}{\partial p_{i}} \quad \text { and } \quad b_{i x}:=\frac{\partial b_{i}(\cdot)}{\partial X_{i}} .
$$

Rearranging equations (6) and (7) with respect to stock-price derivatives we obtain:

$$
\left(\frac{\partial^{2} \tilde{\pi}}{\partial X_{1}^{2}}-\delta b_{1 x}\right) \frac{\partial X_{1}^{*}}{\partial p_{1}}+\frac{\partial^{2} \tilde{\pi}}{\partial X_{1} \partial X_{2}} \frac{\partial X_{2}^{*}}{\partial p_{1}}=\delta b_{1 p}-\frac{\partial^{2} \tilde{\pi}}{\partial X_{1} \partial p_{1}}
$$

\footnotetext{
${ }^{2}$ Following Flaaten (1991), we make no attempt to obtain the complete solution to the problem and limit the focus of our study to the steady-state solution. The general optimal approach path to the steady state is not known (Clark 1976, p. 323).

${ }^{3}$ Necessary conditions are also sufficient for optimality if total profits are concave in $X_{1}$ and $X_{2}$.
} 


$$
\frac{\partial^{2} \tilde{\pi}}{\partial X_{2} \partial X_{1}} \frac{\partial X_{1}^{*}}{\partial p_{1}}+\left(\frac{\partial^{2} \tilde{\pi}}{\partial X_{2}^{2}}-\delta b_{2 x}\right) \frac{\partial X_{2}^{*}}{\partial p_{1}}=-\frac{\partial^{2} \tilde{\pi}}{\partial X_{2} \partial p_{1}} .
$$

Applying Cramer's rule, equations (9) and (10) can be solved with respect to the stock-price derivatives, yielding the following equations:

$$
\begin{aligned}
\frac{\partial X_{1}^{*}}{\partial p_{1}} & =\frac{\left(\delta b_{1 p}-\frac{\partial^{2} \tilde{\pi}}{\partial X_{1} \partial p_{1}}\right)\left(\frac{\partial^{2} \tilde{\pi}}{\partial X_{2}^{2}}-\delta b_{2 x}\right)+\frac{\partial^{2} \tilde{\pi}}{\partial X_{1} \partial X_{2}} \frac{\partial^{2} \tilde{\pi}}{\partial X_{1} \partial p_{1}}}{|D|} \\
\frac{\partial X_{2}^{*}}{\partial p_{1}} & =\frac{\left(\delta b_{1 x}-\frac{\partial^{2} \tilde{\pi}}{\partial X_{1}^{2}}\right) \frac{\partial^{2} \tilde{\pi}}{\partial X_{2} \partial p_{1}}+\frac{\partial^{2} \tilde{\pi}}{\partial X_{2} \partial X_{1}}\left(\frac{\partial^{2} \tilde{\pi}}{\partial X_{1} \partial p_{1}}-\delta b_{1 p}\right)}{|D|},
\end{aligned}
$$

where:

$$
|D|=\left|\begin{array}{cc}
\frac{\partial^{2} \tilde{\pi}}{\partial X_{1}^{2}}-\delta b_{1 x} & \frac{\partial^{2} \tilde{\pi}}{\partial X_{1} X_{2}} \\
\frac{\partial^{2} \tilde{\pi}}{\partial X_{2} X_{1}} & \frac{\partial^{2} \tilde{\pi}}{\partial X_{2}^{2}}-\delta b_{2 x}
\end{array}\right| .
$$

However, Flaaten (1991) computes:

$$
\frac{\partial X_{1}^{*}}{\partial p_{1}}=\frac{\delta b_{1 p}\left(\partial^{2} \pi / \partial X_{2}^{2}-\delta b_{2 x}\right)}{|D|}
$$

and

$$
\frac{\partial X_{2}^{*}}{\partial p_{1}}=\frac{-\delta b_{1 p}\left(\partial^{2} \pi / \partial X_{2} \partial X_{1}\right)}{|D|} .
$$

Hence, equations (11) and (12) coincide with Flaaten's computations if and only if $\partial^{2} \tilde{\pi} / \partial X_{i} \partial p_{1}=0,(i=1,2)$. Since $\partial^{2} \tilde{\pi} / \partial X_{1} \partial p_{1}=G_{11}\left(X_{1}^{*}, X_{2}^{*}\right)=r_{1}\left(1-2 X_{1}^{*}-\alpha_{1} X_{2}^{*}\right)$ and $\partial^{2} \tilde{\pi} / \partial X_{2} \partial p_{1}=G_{12}\left(X_{1}^{*}, X_{2}^{*}\right)=\alpha_{1} r_{1} X_{1}^{*}<0$ are different from zero, the proofs to Theorems 5 and 6 in Flaaten (1991) are incorrect. In fact, we can find an illustrative example which reverses Theorem 5, as the following observation reveals.

OBSERVATION: If harvesting in the competing species model (with $\alpha_{i}>0$, $i=1,2)$ is costless, the following holds: $\left(\partial X_{i}^{*} / \partial p_{i}\right) \geq 0$, and $\left(\partial X_{j}^{*} / \partial p_{i}\right) \leq 0$.

As shown in Flaaten (1991), it follows from the second-order condition for the existence of an interior solution that the denominator to both equations (11) and (12) is positive; i.e., $|D|>0$, and the second derivative of the profit function with respect to the own stock, $\partial^{2} \tilde{\pi} / \partial X_{i}^{2}=-2 r_{i} p_{i}$, is negative. Note that $b_{i p}=1$, and $c_{i}=0(i=1,2)$ implies $b_{i x}=0$. Taking into account $\partial^{2} \tilde{\pi} / \partial X_{i} \partial X_{j}=-\alpha_{1} r_{1} p_{1}-\alpha_{2} r_{r} p_{2}<0$ and $G_{i j}\left(X_{1}^{*}\right.$, 
$\left.X_{2}^{*}\right)<0$, the proof reduces thus to show that the marginal growth rate in the steady state exceeds the interest rate; i.e., $G_{i i}\left(X_{1}^{*}, X_{2}^{*}\right) \geq \delta$. Since we assume an interior solution, this is trivially satisfied. To see this, note that the left-hand side of equation (4) will be always greater than the right-hand side, since $G_{j i}=-r_{j} a_{j} X_{j}<0$. That is, the total profit of the marginal unit extracted from the stock would exceed the present value of all future losses that result through harvesting from a lower stock. Therefore, if the interest rate is greater than the marginal growth rate, the optimal management decision is to extinguish the species. At the corner solution; i.e., if one stock size is zero, the stock-price derivatives are zero. If both stocks are positive, the inequalities are strict: A higher economic value of a unit species 1 implies that the stock of species 1 is increased in the steady state, while the stock of species 2 is decreased. In other words, if there are no extraction costs and 1's price increases, the planner would care less about the spillovers from species 1 to species 2 and more about the spillovers that affect species 1 .

As pointed out above, Flaaten (1991) uses the same proof to establish his Theorems 5 and 6 . Thus, the proof to Theorem 6 is also incorrect. This theorem states that the steady-state stock level of one species is: $(i)$ increasing in own harvesting costs and (ii) decreasing in the harvesting costs of competing species. In a supplement to this paper, we numerically show that the part (ii) of Theorem 6 is incorrect. ${ }^{4}$

\section{Summary}

In this paper, we have commented that two essential theorems of Flaaten's (1991) paper on optimal management of competing species are wrong. These theorems make a claim about how the equilibrium stock in the sole owner fishery moves when prices or costs are changed. In contrast to these theorems, we have shown that a higher price of one species does not necessarily imply a lower bioeconomic equilibrium stock. Particularly in the case of costless harvesting, where the theorem is reversed, the optimal resource management decision induces more extraction of the competing species in order to enhance the stock of the more valuable species.

\section{References}

Clark, C. 1976. Mathematical Bioeconomics. New York: John Wiley \& Sons.

Flaaten, O. 1991. Bioeconomics of Sustainable Harvest of Competing Species. Journal of Environmental Economic and Management 20:163-80.

Gause, G.F. 1934 (reprinted 1969). The Struggle for Existence. New York/London: Hafner.

${ }^{4}$ The supplement is available from the authors upon request. 